\title{
Interplay between Near-Field Properties and Au Nanorod Cluster Structure: Extending Hot Spots for Surface-Enhanced Raman Scattering
}

\author{
Klester S. Souza, ${ }^{a, b}$ Erico Teixeira-Neto, ${ }^{c}$ Marcia L. A. Temperini ${ }^{*, a}$ and \\ Diego P. dos Santos ${ }^{\circledR} * d$ \\ ${ }^{a}$ Departamento de Química Fundamental, Universidade de São Paulo, \\ 05508-000 São Paulo-SP, Brazil \\ ${ }^{b}$ Departamento de Química Inorgânica, Instituto de Química, \\ Universidade Federal do Rio Grande do Sul, 91501-970 Porto Alegre-RS, Brazil \\ 'Departamento de Química Inorgânica, Instituto de Química, \\ Universidade Estadual de Campinas, 13083-970 Campinas-SP, Brazil \\ ${ }^{d}$ Departamento de Físico-Química, Instituto de Química, \\ Universidade Estadual de Campinas, 13083-970 Campinas-SP, Brazil
}

\begin{abstract}
Materials science has observed a continuous increase in the use of metal nanoparticles in a wide range of studies, from fundamental physics to technological applications such as photocatalysis and optical communication devices. This broad scope has the same fundamental origin, the localized surface plasmons, whose excitation leads to strong light confinement, especially in the vicinity of closely spaced nanoparticles, the hot spots. The field amplification may be used to amplify the Raman scattering of adsorbed molecules, which is known as surface-enhanced Raman scattering (SERS). A crucial and limiting characteristic of SERS hot spots is their very localized nature, that influences the SERS intensity reproducibility as well as the probabilities of observation of single-molecule SERS signals. In this paper we discuss the correlation between SERS performance and gold nanorod cluster structures using transmission electron microscopy, SERS spectra and numerical simulations. The experimental data showed interesting behavior for the combination of end-to-end and side-by-side interactions, revealing the possibility of creating strong hot spots with a more extended spatial distribution. The results give insights into the development of high-performance SERS substrates.
\end{abstract}

Keywords: SERS, gold nanorod, Fano resonance, hot spot morphology

\section{Introduction}

In the last decade, a research field known as plasmonics has gaining increased attention due to its unique properties. ${ }^{1}$ Plasmons are coherent oscillations modes of electron densities in materials with free-electron response to an incoming excitation (e.g., by electromagnetic radiation). Materials as metals present such coherent oscillations, and if the metal have a nanometric size, the modes become very localized around the nanoparticle surface. The excitation of this localized surface plasmon leads to strong light confinement at nanoscale dimensions, which permits a variety of applications such as biotechnology, ${ }^{2}$ biosensing, ${ }^{3}$ solar energy, ${ }^{4}$ and photocatalysis, ${ }^{5}$ to name a few.

*e-mail: mlatempe@iq.usp.br; santosdp@unicamp.br
One of the main applications of plasmonic nanoparticles is related to the surface-enhanced Raman spectroscopy (SERS). Since its discovered in 1970s, SERS have been established as a powerful technique that allows normally weak Raman signals to be enhanced by up to eight orders of magnitude. Due to its rich vibrational spectroscopic information of a molecule in close proximity to the nanoparticle surface, SERS has been considered a robust analytical sensing technique. The large field enhancement is highly dependent on the metal nanoparticle shape, size and degree of aggregation (cluster morphology). The electromagnetic field in nanoparticle junctions, called hot spot, are responsible for most of the SERS signals even though hot spots are very small compared to the nanostructure volume.$^{6-9} \mathrm{It}$ is the existence of such special regions that makes possible the 
observation of single-molecule SERS (sm-SERS) spectra, representing the ultimate analytical detection level. Apart from the analytical chemistry applications of sm-SERS, experiments at such conditions may provide fundamental insights into the local field properties derived from the surface plasmon excitation. For instance, by analyzing the anti-Stokes to Stokes intensity ratios, it is possible to extract resonance energies of individual hot spots ${ }^{10}$ and aggregation state of spherical nanoparticles..$^{11}$ Therefore, for both fundamental studies and applications of SERS, a comprehensive understanding of the relationship between nanoparticle/cluster structure and the local field enhancement is a fundamental step.

Van Duyne and co-workers ${ }^{12}$ presented a review on the correlation between structural and plasmonic properties for single (spherical) particle clusters. They showed the challenges in such systems and its importance to improve the understanding in the determination of SERS enhancement, controlled manipulation of single nanoparticles and quantitative structure-localized surface plasmon resonance (LSPR) activity relationship. ${ }^{12}$

Gold nanorods (AuNRs) are one of the most studied plasmonic nanostructures due to its broadly tunable aspectratio, which directly affects its surface plasmon resonance. ${ }^{6}$ The large surface curvature associated to the nanoparticle structure produce very large (and very localized) SERS enhancement factors (ratio between the SERS and normal Raman intensities for a single-molecule, a measure of the nanoparticle efficiency in increasing the Raman signal of an adsorbed molecule). This strong field localization may lead to large SERS fluctuations at low concentrations, reducing the probability density of observing large SERS enhancement events. ${ }^{13,14}$

In closely spaced nanoparticles (such as in AuNR clusters), the individual surface plasmon modes of adjacent nanoparticles interact, producing hybridized bonding and antibonding plasmon modes..$^{11}$ Lee et al. ${ }^{15}$ showed that by controlling the morphology of AuNRs clusters, the observed plasmonic response is dependent on the AuNRs relative orientation. Their investigations showed that the end-to-end (ee) orientation leads to higher SERS intensities when compared to the side-by-side (ss) in the case of aggregates with only one of those orientations. ${ }^{15,16}$ Its worth pointing out that the experimental conditions in the aforementioned papers induce only one kind of nanoparticle aggregation orientation. However, in simpler SERS experiments where the AuNRs are deposited over a substrate platform without controlling the aggregation scheme, it could be expected both orientations (ee and ss) to be observed in very distinct structures with possible reduced symmetries. Therefore, how could heterogeneity interfere in the measured SERS intensity? Or how could heterogeneity give us some insight on a better plasmonic SERS substrate for different applications?

Here, we discuss the correlation between SERS intensities and small clusters geometries of AuNRs using transmission electron microscopy (TEM), SERS spectra and classical electrodynamic simulations. Based on the results, it is made a discussion about the impact of the hot spot morphology in the overall SERS enhancement, as well as on the local field enhancement spatial distribution. It is believed that the results presented in this paper may give hints in the search of efficient SERS substrates.

\section{Experimental}

\section{Reagents}

Hexadecyltrimethylammoniumbromide (CTAB) 98\%, L-ascorbic acid, $p$-aminobenzenothiol ( $p$ ABT) and sodium nitrate were purchased from Sigma-Aldrich (USA). Sodium borohydride (99\%) was purchased from Fluka (USA). Deionized water $(18 \mathrm{M} \Omega \mathrm{cm})$ was used in all experiments.

\section{Synthesis of gold nanorods}

Gold nanorods (AuNR) were prepared by the "seed mediated growth method" proposed by Nikoobakht and El-Sayed ${ }^{6}$ with some alterations. Briefly, seed nanoparticles (NP) were prepared by mixing $5 \mathrm{~mL}$ of CTAB solution $\left(0.20 \mathrm{~mol} \mathrm{~L}^{-1}\right)$ with $5.0 \mathrm{~mL}$ of $0.00050 \mathrm{~mol} \mathrm{~L}^{-1} \mathrm{HAuCl}_{4}$. To the stirred solution, $0.60 \mathrm{~mL}$ of ice-cold $0.010 \mathrm{~mol} \mathrm{~L}^{-1}$ $\mathrm{NaBH}_{4}$ was added, which resulted in the formation of a brownish yellow solution. Vigorous stirring of the seed solution continued for $2 \mathrm{~min}$ and then the solution was kept at $25^{\circ} \mathrm{C}$ for $5 \mathrm{~min}$ prior to use. For the growth NPs, $5 \mathrm{~mL}$ of CTAB solution $\left(0.20 \mathrm{~mol} \mathrm{~L}^{-1}\right)$ was added to $0.20 \mathrm{~mL}$ of $0.0040 \mathrm{~mol} \mathrm{~L}^{-1} \mathrm{AgNO}_{3}$. To this solution, $5.0 \mathrm{~mL}$ of $0.0010 \mathrm{~mol} \mathrm{~L}^{-1} \mathrm{HAuCl}_{4}$ was added, and after gentle mixing of the solution $70 \mu \mathrm{L}$ of $0.0788 \mathrm{~mol} \mathrm{~L}^{-1}$ ascorbic acid was added. Ascorbic acid as a mild reducing agent changes the growth solution from dark yellow to colorless. The final step was the addition of $12 \mu \mathrm{L}$ of the seed solution to the growth solution at $30{ }^{\circ} \mathrm{C}$ for $10 \mathrm{~min}$. For stop the growth of the NPs and stabilized the AuNRs was added to the final solution $500 \mu \mathrm{L}$ of $p$-aminobenzenothiol ( $p$ ABT) $1 \mathrm{mmol} \mathrm{L}^{-1}$ to exchange the CTAB ligands attached to the nanoparticles (NPs) surface with $p$ ABT. After that, the colloidal solution was purified by three centrifugation cycles $(10,000 \mathrm{rpm}$ for $15 \mathrm{~min}$ ) in a $3: 1$ acetonitrile/water solution with the final cycle in water. 


\section{Characterization}

Extinction spectra of colloidal aqueous suspension of AuNR was carried out using a Shimadzu UVPC-3101. TEM images were performed on the TEM Jeol JEM 2100 on bright field mode with Parallel Illumination. FT-Raman spectra were recorded in a FT-Raman Bruker RFS 100 spectrometer with a liquid nitrogen cooled germanium detector, and $1064 \mathrm{~nm}$ excitation radiation (Nd:YAG laser, Coherent Compass 1064-500N). The spectra were obtained at $150 \mu \mathrm{W}$ and accumulation of 512 scans. The SERS spectra were obtained in a Renishaw InVia coupled to a Leica DM2500M microscope, with a diode laser emission at $785 \mathrm{~nm}$; laser power remained at ca. $1 \mathrm{~mW}$ during SERS measurements. The SERS spectra were measured using a $100 \times$ objective (numerical aperture, NA, of 0.9) with an exposure time of $10 \mathrm{~s}$.

The as-prepared colloidal aqueous solution of gold nanorods, AuNR functionalized with $p$ ABT (AuNRs@pABT) was deposited in the carbon grid type B 400 mesh purchased from Ted Pella, INC. This substrate was used for both TEM images and SERS spectra give us a way to identify the area of the surface where the SERS analysis were made. The SERS spectra were obtained in a Renishaw InVia system spectrometer coupled to a Leica microscope. The laser excitation and power in the sample were $785 \mathrm{~nm}$ (Renishaw solid-state laser model HPNIR785) and ca. $17 \mu \mathrm{W}$, respectively. Spectra were measured using a $100 \times$ objective $(\mathrm{NA}=0.9)$ with an exposure time of $10 \mathrm{~s}$ and 1 scan.

For correlating SERS spectra and TEM images of the aggregate morphologies, we first obtained the SERS spectra by mapping the surface in line between visible spots seen through the optical microscopy. Then, we correlated the SERS spectra and TEM images through their same distance from the visible spots (see Figure S1, Supplementary Information (SI) section).

\section{Electrodynamics simulations}

The optical properties of AuNR clusters were simulated by the discrete dipole approximation (DDA) methodology, using the DDSCAT program. ${ }^{17}$ In short, in this method the target structure is represented in terms of a cubic lattice of polarizable point sources. The incident electromagnetic field induces a dipole in each location of the target, which acts as source of electric field to the neighbor dipoles. The convergence of this system of coupled equations representing the interaction of the target with incident radiation permits the calculation of optical parameters such as the extinction, scattering and absorption cross-sections (or coefficients - ratio between the process and geometrical cross-sections) as function of incident light frequency. The simulations were performed with grid spacings equal to $0.25 \mathrm{~nm}$ (small clusters, up to 3 nanoparticles) and $0.5 \mathrm{~nm}$ (larger clusters), conditions that ensure proper near-field and scattering convergences ${ }^{18}$ (see Figure S2, SI section).

The frequency-dependent target polarization is calculated in terms of the material optical response described by the dielectric function. In all simulations it was considered the experimental dielectric function of gold. ${ }^{19}$ The calculation of the expected SERS enhancement factors (EF) due to the plasmon excitation was performed by the $\mathrm{E}^{4}$ approximation, where the SERS enhancement is described by the fourth power of the local electric field enhancement. ${ }^{20}$ The DDA simulations were also compared with boundary element method (BEM) simulations for the case of small cluster (up to 3 nanoparticles), in which only the surfaces that separate the different media must be discretized. This method is based on the calculation of surface charges and currents in each surface element, from which the crosssections and local electric can be computed. The BEM method was used for the interpretation of the plasmonic modes in the AuNRs clusters. All simulations were performed with the MNPBEM17 program and the surface discretization was carried out aiming at a convergence with the DDA scattering spectrum (see SI section). ${ }^{21}$ In all simulations of AuNR clusters, the interparticle surface gap distance was considered as $1 \mathrm{~nm}$.

\section{Results and Discussion}

\section{Near-field properties of AuNRs clusters}

AuNRs geometry and surface chemistry can be exploited to direct the structure of clusters in ss or end-to-end ee configurations. Lee et al. ${ }^{22}$ demonstrated that whereas in the ee aggregation scheme the plasmonic coupling leads to a redshift in the extinction spectrum and to an increase in the local electric field enhancement, in the ss interaction it can be observed a blueshift in the optical response as well as a decrease in the surface average local field amplification. ${ }^{15}$ The key results of the above reports are reproduced in Figure 1, in which we present the DDA simulated extinction spectra (a) for different clusters of AuNRs (aspect ratio 3) in water environment and a spatial distribution of the expected SERS enhancement factors ( $b$ and $c$ ) for clusters with ss and ee configurations. As it can be seen the largest field concentrations are expected in the ee configuration.

The above results show that, for SERS applications, the ee aggregation is a better choice aiming at maximizing the measured intensities. However, the nature of such coupling 

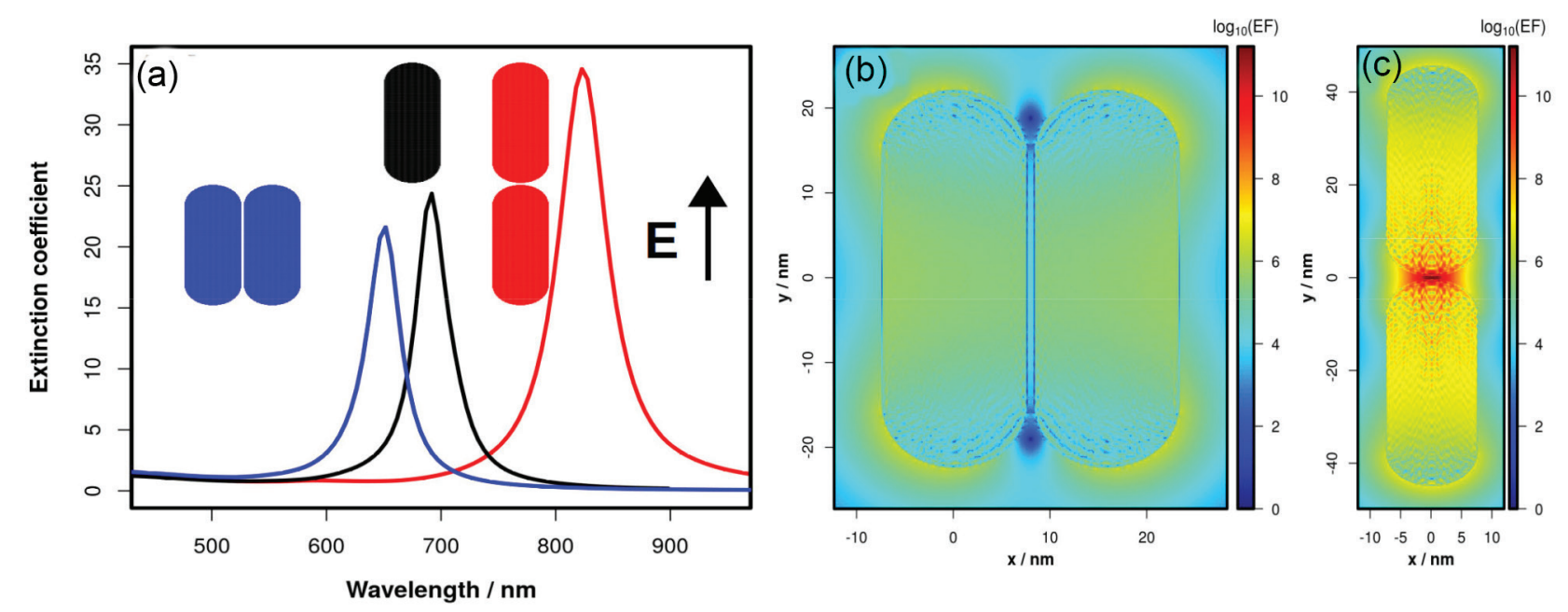

Figure 1. DDA simulated extinction spectra for different clusters of AuNRs (aspect ratio 3) in water environment (a) and a spatial distribution of the expected SERS enhancement factors (b) in logarithmic scale for AuNR dimers in ss (b) and ee (c) configurations. The gap size in all simulations was taken as $1 \mathrm{~nm}$.

produces extremely localized hots pots ${ }^{14}$ at the nanorod tips (Figure 1c), which may lead to strong spatial SERS intensity fluctuations ${ }^{23}$ and reduced probabilities for a single-molecule event to be detected once only a molecule that reaches such small volume locations generates a measurable signal. In this sense, it is reasonable to search for ways to increase the spatial distribution of hots spots over a greater area on the AuNR surface, which necessarily must be accompanied by a field amplification in the ss interaction. To tackle this problem, we investigate the effect of asymmetric structures in the plasmonic coupling of AuNRs. In the case of asymmetrical structures, a special optical response may be observed, the Fano resonance, which is a result of coupling between bright (large scattering cross-section) and dark (low scattering cross-section) modes due to symmetry breaking. ${ }^{24-26}$ The excitation of such Fano resonances may lead to the observation of differential optical responses, as local field and temperature spatial distributions. ${ }^{24,27}$ In this section we investigate, by numerical simulations, the near-field properties of asymmetrical clusters and the possible effect of Fano interferences in the localization of SERS enhancement factors. For that purpose we investigate a simple model system from which we draw conclusions that can be extrapolated to more complex structures that may be observed in experimental conditions.

The Fano resonance is characterized by a non Lorentzian response in the scattering spectra of the nanostructure, due to an out-of-phase interaction between bright and dark modes, promoting a destructive interaction and a decrease in the scattering probability. Usually such dark and bright modes are, respectively, quadrupole and dipole modes. However, we should be able to observe such resonances in the event of interaction between any spectrally overlapping darker and brighter modes, in the sense of scattering crosssections, as long as the two modes are out-of-phase. If we analyze Figure 1 in terms of single particle versus dimer responses, it is possible to observe that the ss interaction leads to a destructive interaction between the two in-phase parallel dipoles in each AuNR plasmon mode. ${ }^{15}$ The ee configuration, on the other hand, leads to a constructive interaction between the plasmon modes. Therefore, we could think about the ss and ee configurations as darker and brighter modes, respectively, and investigate the resulting properties of Fano interferences between them. Figure 2 shows DDA simulations for a cluster comprised by three AuNRs, in which both ee and ss configurations are observed.

The optical response presented in Figure 2 for the asymmetrical trimer structure is very different from the symmetric cases in Figure 1. The plasmonic response is characterized by two main resonances at $662 \mathrm{~nm}$ and $796 \mathrm{~nm}$ with a shoulder at $710 \mathrm{~nm}$. The higher energy mode is also characterized by an asymmetrical response in the scattering spectrum (Figure 2a, inset, blue line), which is indicative of a Fano interference. Also, it can be noticed that such modes are dominated by absorption, i.e., the plasmon relaxation dynamics is essentially non-radiative, which can be very useful in the hot electron generation dynamics for plasmonic photocatalysis. ${ }^{28}$ The above results clearly indicate a complex plasmonic interaction in this asymmetric structure. However a simple classical coupled oscillator mode ${ }^{29}$ can be used to describe most of the observed properties in the simulated scattering spectrum. This analysis can be performed by the classical equations of motion for three coupled dumped harmonic oscillators (each contributing to a resonance in the scattering spectrum): ${ }^{30}$ 

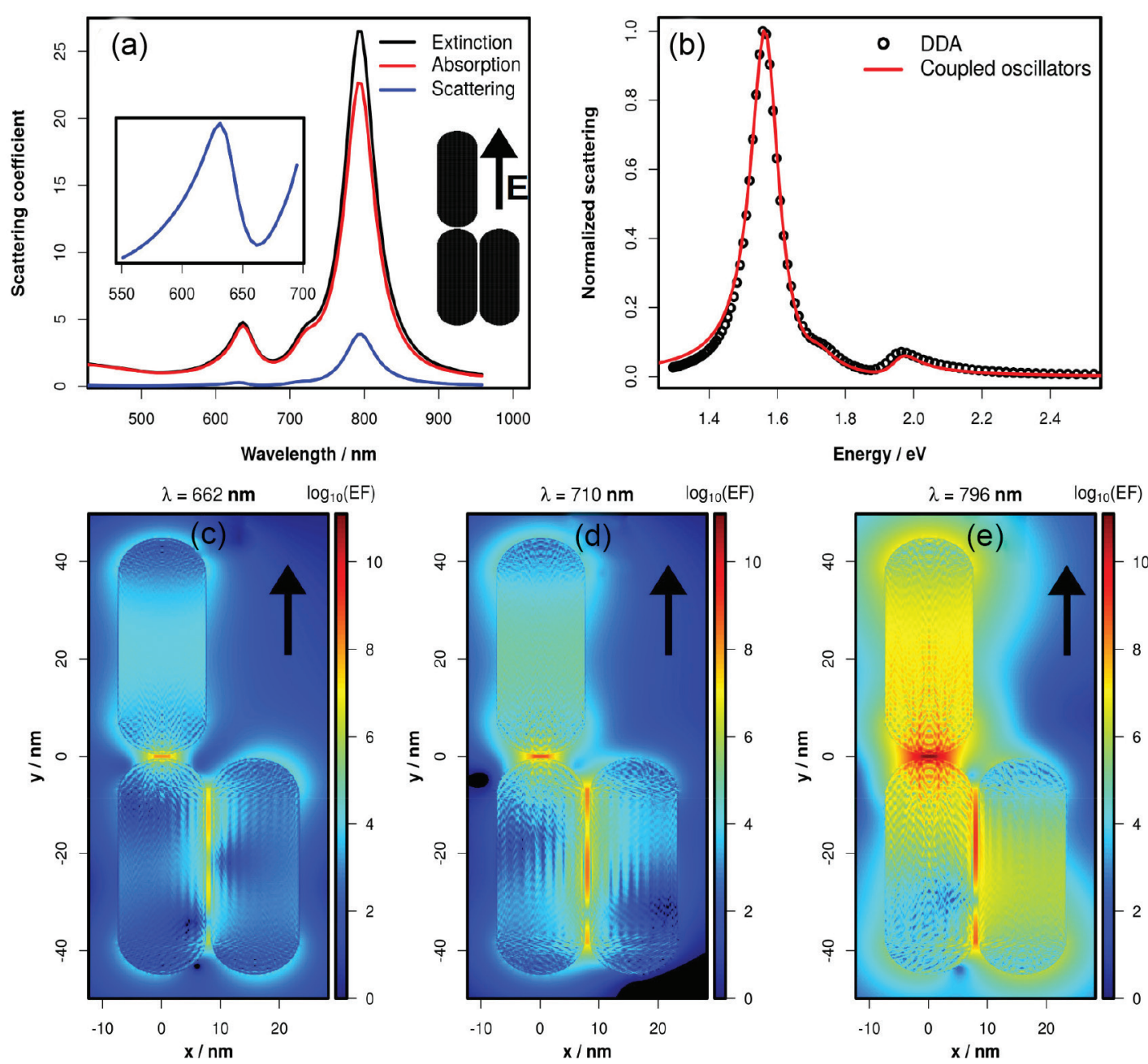

Figure 2. DDA simulations for a cluster comprised by three AuNRs in water: (a) Extinction (black line), absorption (red line) and scattering (blue line) spectra; (b) Comparison between the simulated scattering spectrum and the coupled oscillator model; (c)-(e) Maps of the SERS enhancement factor in logarithmic scale for wavelength excitations at $662 \mathrm{~nm}, 710 \mathrm{~nm}$ and $796 \mathrm{~nm}$, respectively.

$$
\left\{\begin{array}{c}
\ddot{x}_{1}(t)+\gamma_{1} \dot{x}_{1}(t)+\omega_{1}^{2} x_{1}(t)-\chi_{12}^{2} x_{2}(t)-\chi_{13}^{2} x_{3}(t)=A e^{-i \omega t} \\
\ddot{x}_{2}(t)+\gamma_{2} \dot{x}_{2}(t)+\omega_{2}^{2} x_{2}(t)-\chi_{12}^{2} x_{1}(t)-\chi_{23}^{2} x_{3}(t)=0 \\
\ddot{x}_{3}(t)+\gamma_{3} \dot{x}_{3}(t)+\omega_{3}^{2} x_{3}(t)-\chi_{13}^{2} x_{1}(t)-\chi_{23}^{2} x_{2}(t)=0
\end{array}\right\}
$$

where $x_{i}(t)$ is the instantaneous amplitude of displacement of oscillator $i$ whose characteristic frequency and dumping constant are $\omega_{i}$ and $\gamma_{i}$, respectively. Each of the dot symbols above $x_{i}$ corresponds to its time-derivative. $\chi_{i j}$ is a measure of the coupling between oscillators $i \mathrm{e} j$. A solution for the above system of equations can be obtained by a assuming a time dependence for each oscillator in the form:

$$
x_{i}(t)=\mathrm{C}_{i}\left(\omega_{i}\right) e^{-\mathrm{i} \omega \mathrm{t}}
$$

where $\left|\mathrm{C}_{i}\left(\omega_{i}\right)\right|^{2}$ is the squared maximum amplitude for the oscillator and may be used to fit the DDA simulated scattering spectra of Figure $2 b$. The fitting parameters are presented in Table 1.
Table 1. Coupled oscillator model fitting parameters

\begin{tabular}{lcc}
\hline $\begin{array}{l}\text { Resonance } \\
\text { energies / eV }\end{array}$ & $\begin{array}{c}\text { Dumping } \\
\text { constants / eV }\end{array}$ & $\begin{array}{c}\text { Coupling } \\
\text { constants / eV }\end{array}$ \\
\hline$\omega_{1}=1.65$ & $\gamma_{1}=0.1$ & $\chi_{12}=0.4$ \\
$\omega_{2}=1.70$ & $\gamma_{2}=0.1$ & $\chi_{13}=0.7$ \\
$\omega_{3}=1.90$ & $\gamma_{3}=0.1$ & $\chi_{23}=0$ \\
\hline
\end{tabular}

As it can be seen in Figure 2b, a good fit can be observed if we treat the trimer response as a result of a simple interaction between modes with resonance energies close to the three structure of Figure 1, specially for oscillator 3 . Therefore, it is possible to interpret the simulated spectrum as a result of interactions of a single particle resonance with ee and ss dimers resonances. This analysis is in agreement with the simulated BEM surface charge densities presented as suplementary information (Figure S4, SI section). The Fano resonance is due to a phase shift between the single particle and the ss dimer resonances. 
The above coupled oscillator analysis reveals that the ss mode is more affected by ee than by the single particle mode (in fact we set a zero coupling constant in the fitting for this interaction). This suggests that the trimer EF distributions may deviate from the ss field distributions for dimers presented in Figure 1. Figures 2c-e show the EF maps in the a plane that passes through the three AuNRs centers. The maps were calculated for incident light wavelengths at $662 \mathrm{~nm}$ (Fano dip, maximum interference), $710 \mathrm{~nm}$ (shoulder in the scattering spectra) and $796 \mathrm{~nm}$.

A substantial field enhancement can be observed in the vicinity of ss interacting structures. The maps show a general behavior of strong EF localized between the ss interacting particles, which decay exponentially as we move farther from the ee hot spot, the main difference among the three excitations being related to the magnitude of enhancements. DDA simulations with a perpendicular polarization (i.e., along the AuNR transversal mode, presented in Figure S5, SI section) leads to considerable lower EFs in this region. These results suggest that a significant contribution for the observation of non-destructive interference in the region between ss interacting particles is due to the strong polarization created by the ee hot spot. Also, it can be observed regions of maximum and minimum field enhancement (see Figures S2, S5 and S6, SI section), which may be attributed to partial destructive interferences.

The above results indicate that asymmetrical AuNRs cluster may present complex plasmonic far-field responses, including the possibility of Fano-like resonances. However, as far as SERS is concerned (near-field properties derived from such excitations), asymmetrical structures with mixed of ee and ss interactions may produce a simpler observation: the field enhancements are not uniquely localized at the AuNRs tips, but may extend to the AuNRs sides with strong field enhancements, which is an interesting characteristic regarding the availability of large SERS EFs for an adsorbing molecule. In the next section we investigate the correlation between the SERS performance and cluster geometry, seeking for an indication that asymmetric structures may hold a key for creating improved platforms for SERS applications.

\section{SERS response of AuNRs substrates}

Figure 3 shows the extinction spectrum of the gold nanorods solution and its TEM image. The spectrum shows
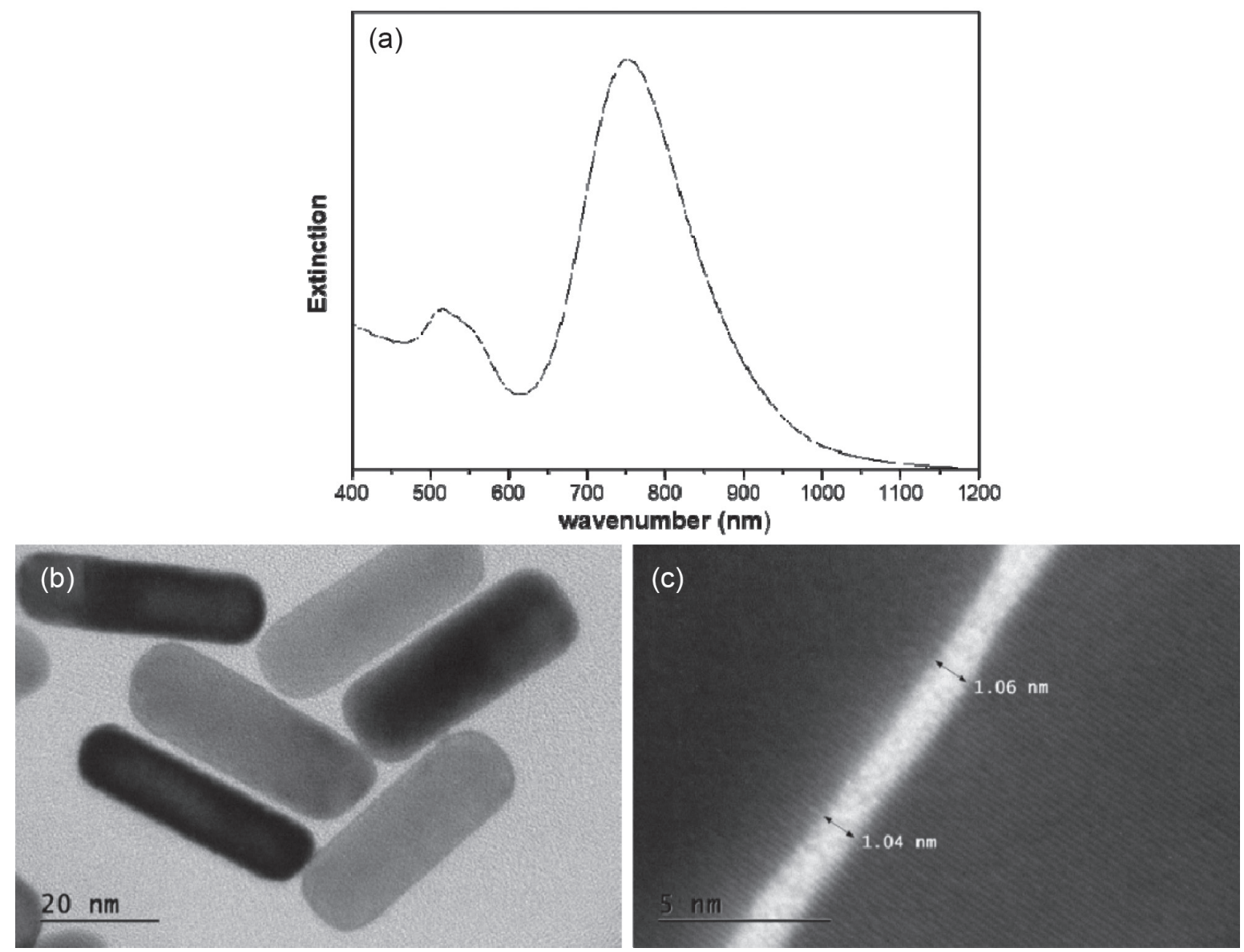

Figure 3. (a) Extinction spectra of the colloidal suspension of AuNR; (b) TEM images of the AuNR and (c) image of the interparticle region between AuNRs. 
a band at wavelength around $520 \mathrm{~nm}$ corresponding to the transverse plasmon oscillations and a higher intensity broad band around $780 \mathrm{~nm}$ corresponding to the longitudinal plasmon oscillations. The AuNR presented an average aspect ratio of $3.0(48 \pm 4 \mathrm{~nm}$ and $16 \pm 2 \mathrm{~nm})$ obtained by counting over 500 particles on TEM images. The redshift observed in respect to the simulation of Figure 1a may be attributed to the particle size/shape dispersion as well as to the CTAB layer contribution to the effective refractive index of the AuNR surrounding medium. Figure $3 \mathrm{c}$ shows a high resolution TEM image from which it is possible to observe a gap between the AuNRs surfaces of ca. $1 \mathrm{~nm}$. This gap size was, therefore, used in all DDA simulations in this manuscript as the minimum distance between AuNRs surfaces.

In Figure 4, normal Raman and SERS spectra, of the $p \mathrm{ABT}$, for different substrates are presented. $p \mathrm{ABT}$ have been the reason of a large debate in the literature due to the Raman behavior in different nanoparticle systems. ${ }^{31-35}$ In this study these queerly is not relevant since we are more interested in the enhancement behavior of the band at $1075 \mathrm{~cm}^{-1} \mathrm{v}(\mathrm{CS})$ that is not related to the charge transfer process. $^{31}$

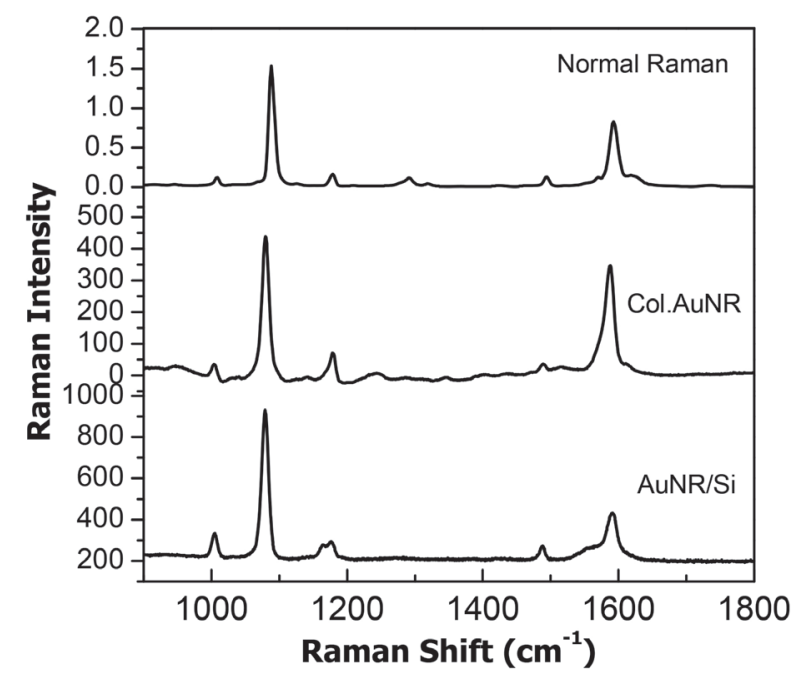

Figure 4. Raman spectra of solid $p \mathrm{ABT}$ and SERS spectra of $p \mathrm{ABT}$ on gold nanorods (AuNR) colloidal solution and on AuNR multilayer deposited on Si wafer.

Since the band at $1075 \mathrm{~cm}^{-1}$ do not suffer intensity variations due to charge transfer, the changes in intensity will arise only, or predominantly so, due to electromagnetic effects from the plasmon excitation.

To analyze the relationship between morphology of AuNR clusters and the SERS intensities we performed SERS analysis on a substrate containing only single aggregates of AuNR. Figure 5 shows the SERS normalized intensity distribution for $p$ ABT band at ca. $1075 \mathrm{~cm}^{-1}$. The data for the histogram was obtained from SERS mapping measurements of an area of $1 \mu \mathrm{m} \times 1 \mu \mathrm{m}$.

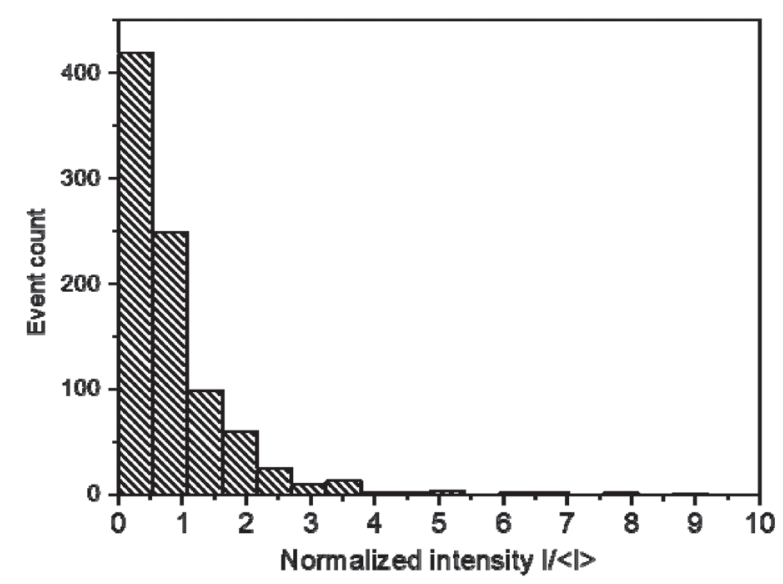

Figure 5. SERS intensity histogram for $p$ ABT adsorbed on AuNR aggregates. 1000 spectra, obtained sequentially scanning the substrate surface, were used to generate the histogram.

The histograms show a tailed distribution of SERS intensities, with a large number of zero-intensity events and bursts of intensities up to 10 times the average value. This behavior can be associated with a small number of aggregates within the illumination area of the laser beam. The large intensity variation is an indicative of a distribution of clusters with a variety of geometries and number of particles. Therefore, a complete picture of the structureactivity relationship for this substrate is not possible and we keep our discussion for a few structures.

Figure 6 presents a correlation between TEM image and $p$ ABT SERS spectrum originated from each structure.

The selected structures in Figure 6 show very distinct geometries. Cluster 1 presents the lowest number of particles in the sequence, which progressively increases in clusters 2 and 3. On the other hand, cluster 2 presents the largest sequence of ee arranged AuNRs (with three particles), whereas for clusters 1 and 3, it can be observed a greater contribution of ss arrangement. In the case of cluster 3 it can also be observed T-shape like arrangements of AuNRs, which do not contribute with very strong enhancements as compared to the ee arrangement, as it will be shown below.

Considering the shape structure and number of particles in each cluster, it could be possible to suggest that the structure with largest number of particles and ee arrangements would provide the largest SERS enhancements. In this regard, it should be expected cluster 2 to present largest SERS intensities for $p A B T$ molecule than cluster 1 . However, this is not observed in the experimental SERS spectra. In fact, the spectra from the two clusters are very similar in terms of measured 

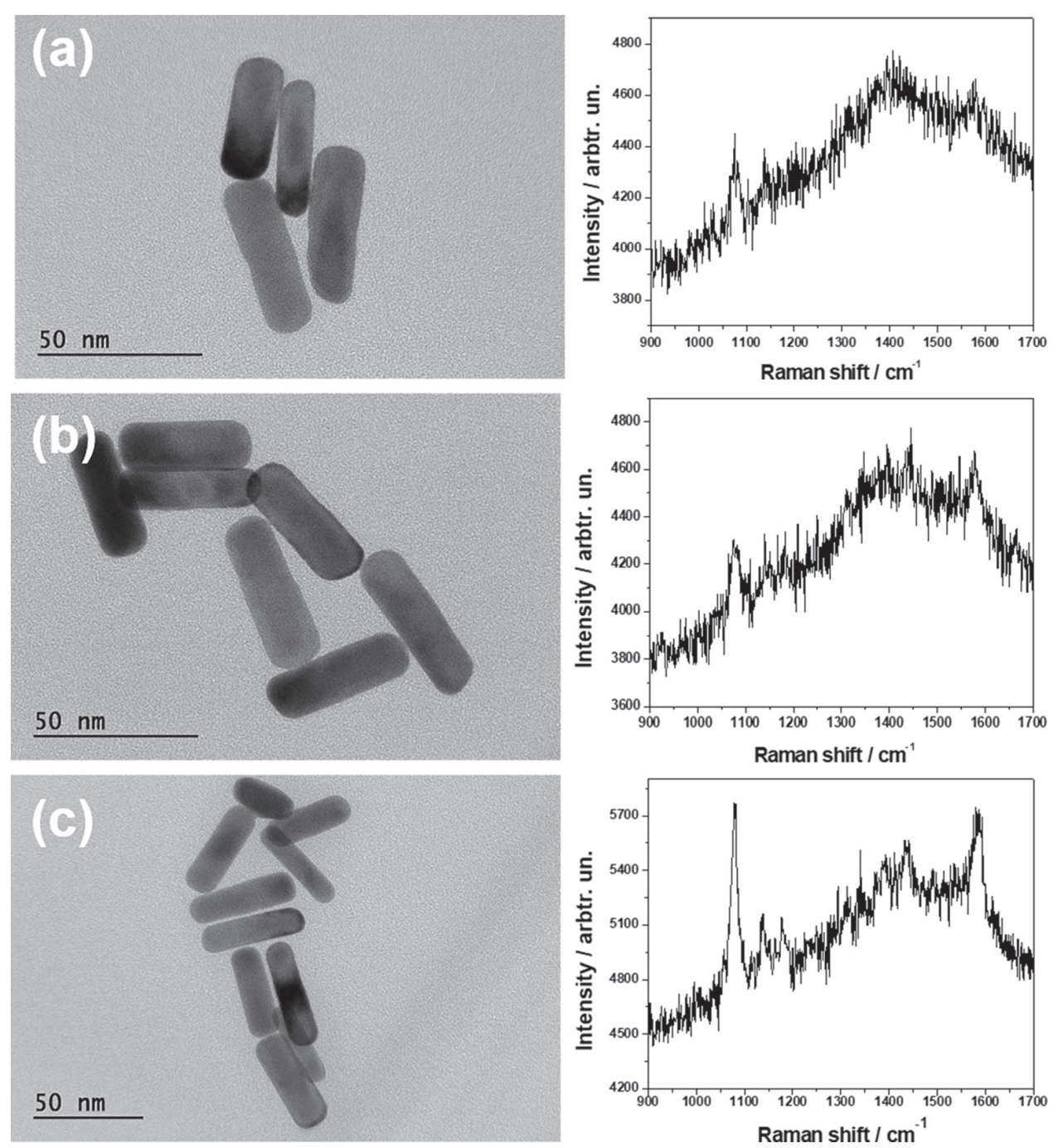

Figure 6. Correlated TEM images and SERS measurements of AuNR aggregates. (a) cluster 1; (b) cluster 2 and (c) cluster 3. In the right side of the figure is presented the SERS spectrum for each AuNR cluster.

intensities. This is an interesting result, since cluster 1 shows a greater contribution of ss arrangement of AuNRs compared to cluster 2 . This result seems to be in accordance with the observed in the previous section regarding the effect of structural asymmetry in the local field distribution. Cluster 3, in a similar way as cluster 1, presents regions of ee coupling and a considerable contribution of ss arrangements, however with a greater number of particles as compared to cluster 1 . This is reflected in the experimental SERS intensities.

The above results suggest that the union of ee and ss configurations in a given cluster may lead to considerable SERS efficiencies for a given analyte molecule, maybe even better than structures with only ee arrangements. This is in accordance with the results for the Fano interaction in the model trimer in the previous section. To analyze the possibility of observation of considerable field enhancements in the vicinity of ss AuNRs, enhancement factor simulations by the DDA method were performed for clusters 1 and 3. The results are presented in Figure 7.

As it can be seen in Figure 7, the enhancement factor simulation shows a considerable distribution of large field enhancements in the vicinity of ee (as expected) and ss arranged AuNRs, which may be due to Fanolike interferences and/or to the strong field polarization promoted by the ee hot spot. In the case of cluster 3, it is possible to observe greater (or at least similar) enhancements (with large spatial distribution) in the ss hot spots than in the hot spots generated by the T-shaped arrangements. The above results and the discussion in the previous section point to the possibility of creating very efficient SERS hot spots by nanoparticle aggregation engineering aiming at maximizing hot spot strength and spatial distribution, which would contribute to large SERS intensities with better signal reproducibility and larger probabilities for single-molecule detection. 

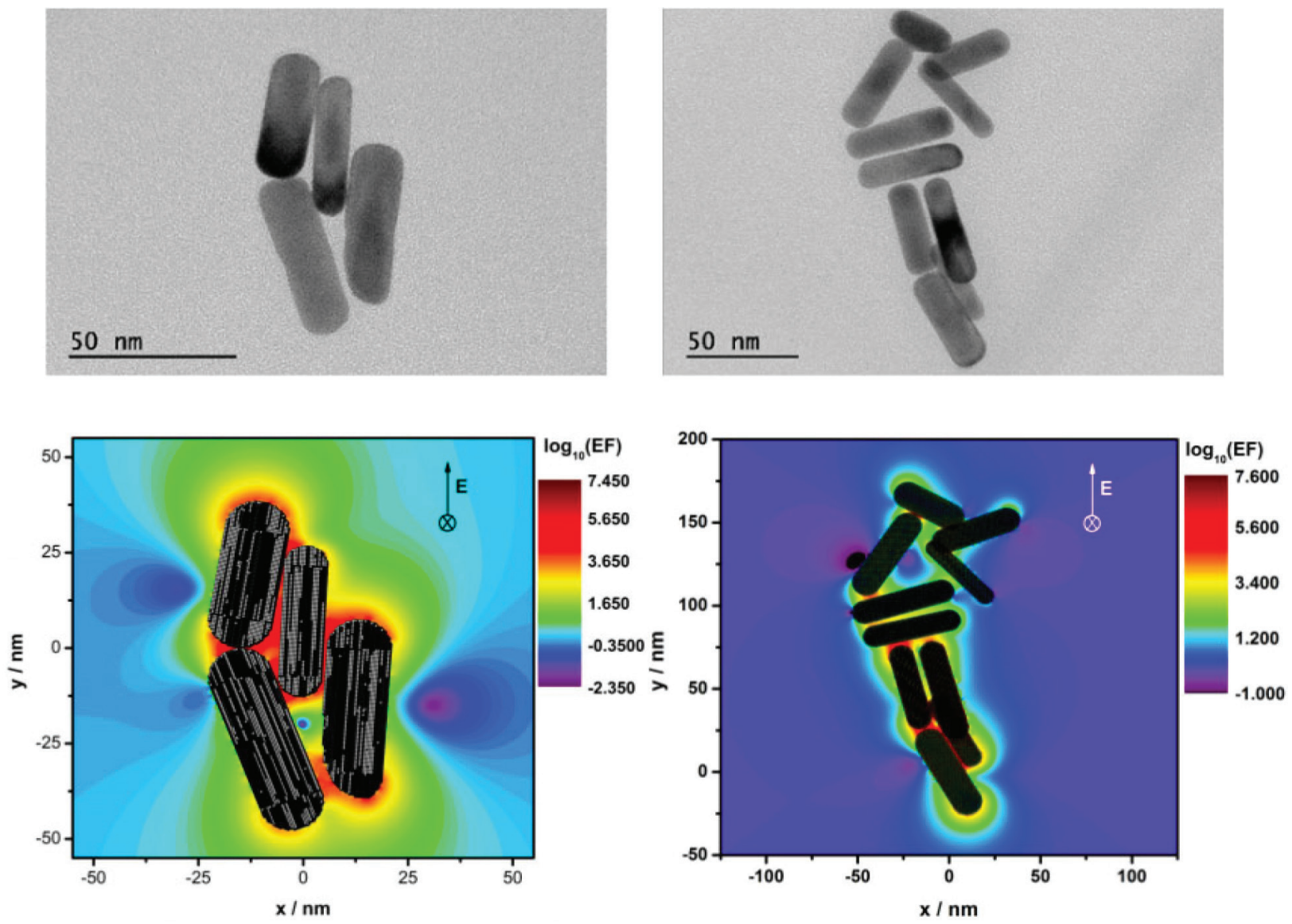

Figure 7. Local electric field enhancement factor maps produced via discrete dipole approximation (DDA) simulation, the aggregates simulated are those which we obtained the SERS spectra. Laser excitation $785 \mathrm{~nm}$.

\section{Conclusions}

In summary, synthesis and characterization of gold nanorods (AuNR), DDA simulation and SERS spectra of small AuNR aggregate were obtained. Spectral and structural characterization, of the AuNR clusters, was accomplished by correlating SERS spectra and transmission electron microscopy.

DDA simulations suggested that asymmetrical structures, those having a mixture of side-by-side (ss) and end-to-end (ee) interaction in AuNR clusters, may present strong enhancements in the vicinity of ss interacting particles which may increase the SERS performance if compared to the extremely localized nature of ee clusters. The experimental results somehow validated that the combination of ee and ss configurations in a given cluster leads to a considerable SERS efficiency for a given analyte molecule, maybe even better than structures with only ee arrangements. It seems that a combination of individuals plasmon modes (bright and dark modes) in the AuNR clusters are contributing for such unusual behavior. Besides that, the discussion presented in this paper gives us insights about an ideal nanoparticle system for fabricating a substrate with a high SERS efficiency.

\section{Supplementary Information}

Supplementary Information (with details about the SERS measurement procedure and further DDA and BEM simulations regarding incident light polarization direction and surface charge densities) is available free of charge at http://jbcs.sbq.org.br as a PDF file.

\section{Acknowledgments}

The authors thank the FAPESP project No. 2011/17923-9 and No. 2016/21070-5. MLAT thanks to CNPq fellowship (302792/2015-5). DPS also thanks to CNPq (408985/2016-0). The authors thank to CA of IQUSP where the TEM images were obtained. This study was financed in part by CAPES - Finance Code 001. This work used resources of the Centro Nacional de Processamento de Alto Desempenho em São Paulo (CENAPAD-SP). 


\section{References}

1. Jiang, N.; Zhuo, X.; Wang, J.; Chem. Rev. 2017, 118, 3054.

2. Lim, W. Q.; Gao, Z.; Nano Today 2016, 11, 168.

3. Szekeres, G. P.; Kneipp, J.; Front. Chem. 2019, 7, DOI: 10.3389/ fchem.2019.00030.

4. Li, J.; Cushing, S. K.; Meng, F.; Senty, T. R.; Bristow, A. D.; Wu, N.; Nat. Photonics 2015, 9, 601.

5. Zhou, L.; Swearer, D. F.; Zhang, C.; Robatjazi, H.; Zhao, H.; Henderson, L.; Dong, L.; Christopher, P.; Carter, E. A.; Nordlander, P.; Halas, N. J.; Science 2018, 362, 69.

6. Nikoobakht, B.; El-Sayed, M.; Chem. Mater. 2003, 16, 1957.

7. Chen, G.; Wang, Y.; Yang, M.; Xu, J.; Goh, S. J.; Pan, M.; Chen, H.; J. Am. Chem. Soc. 2010, 132, 3644.

8. Lee, S. Y.; Hung, L.; Lang, G. S.; Cornett, J. E.; Mayergoyz, I. D.; Rabin, O.; ACS Nano 2010, 4, 5763.

9. Le Ru, E. C.; Grand, J.; Sow, I.; Somerville, W. R. C.; Etchegoin, P. G.; Treguer-Delapierre, M.; Charron, G.; Félidj, N.; Lévi, G.; Aubard, J.; Nano Lett. 2011, 11, 5013.

10. dos Santos, D. P.; Temperini, M. L. A.; Brolo, A. G.; J. Am. Chem. Soc. 2012, 134, 13492.

11. dos Santos, D. P.; Temperini, M. L. A.; Brolo, A. G.; J. Phys. Chem. C 2016, 120, 20877.

12. Kleinman, S. L.; Sharma, B.; Blaber, M. G.; Henry, A. I.; Valley, N.; Freeman, R. G.; Natan, M. J.; Schatz, G. C.; Van Duyne, R. P.; J. Am. Chem. Soc. 2013, 135, 301.

13. Le Ru, E. C.; Etchegoin, P. G.; Meyer, M.; J. Chem. Phys. 2006, 125, 204701.

14. Kie, E. J.; Kie, R. F.; Santos, D. P.; Brolo, A. G.; J. Phys. Chem. C 2017, 121, 25487.

15. Lee, A.; Ahmed, A.; dos Santos, D. P.; Coombs, N.; Park, J. I.; Gordon, R.; Brolo, A. G.; Kumacheva, E.; J. Phys. Chem. C 2012, 116, 5538.

16. Stewart, A. F.; Lee, A.; Ahmed, A.; Ip, S.; Kumacheva, E.; Walker, G. C.; ACS Nano 2014, 8, 5462.
17. Draine, B. T.; Flatau, P. J.; J. Opt. Soc. Am. A 1994, 11, 1491.

18. Hao, E.; Schatz, G. C.; J. Chem. Phys. 2004, 120, 357.

19. Johnson, P. B.; Christy, R. W.; Phys. Rev. B 1972, 6, 4370.

20. Le Ru, E. C.; Etchegoin, P. G.; Chem. Phys. Lett. 2006, 423, 63.

21. Hohenester, U.; Trügler, A.; Comput. Phys. Commun. 2012, 183,370 .

22. Lee, A.; Andrade, G. F. S.; Ahmed, A.; Souza, M. L.; Coombs, N.; Liu, K.; Gordon, R.; Brolo, A. G.; Kumacheva, E.; J. Am. Chem. Soc. 2011, 133, 7563.

23. dos Santos, D. P.; Temperini, M. L. A.; Brolo, A. G.; Acc. Chem. Res. 2019, 52, 456.

24. Zhang, S.; Bao, K.; Halas, N. J.; Xu, H.; Nordlander, P.; Nano Lett. 2011, 11, 1657.

25. Mirin, N. A.; Bao, K.; Nordlander, P.; J. Phys. Chem. A 2009, 113, 4028.

26. Miroshnichenko, A. E.; Flach, S.; Kivshar, Y. S.; Rev. Mod. Phys. 2010, 82, 2257.

27. Baldwin, C. L.; Bigelow, N. W.; Masiello, D. J.; J. Phys. Chem. Lett. 2014, 5, 1347.

28. Cortés, E.; Adv. Opt. Mater. 2017, 5, 1.

29. Joe, Y. S.; Satanin, A. M.; Kim, C. S.; Phys. Scr. 2006, 74, 259.

30. Mukherjee, S.; Sobhani, H.; Lassiter, J. B.; Bardhan, R.; Nordlander, P.; Halas, N. J.; Nano Lett. 2010, 10, 2694.

31. Osawa, M.; Matsuda, N.; Yoshii, K.; Uchida, I.; J. Phys. Chem. 1994, 98, 12702.

32. Kim, K.; Kim, K. L.; Shin, D.; Choi, J.; Shin, K. S.; J. Phys. Chem C 2012, 116, 4774.

33. Kim, K.; Kim, K. L.; Lee, H. B.; Shin, K. S.; J. Phys. Chem. C 2012, 116, 11635.

34. Tian, X.; Chen, L.; Xu, H.; Sun, M.; RSC Adv. 2012, $2,8289$.

35. Choi, H. K.; Shon, H. K.; Yu, H.; Lee, T. G.; Kim, Z. H.; J. Phys. Chem. Lett. 2013, 4, 1079.

Submitted: March 20, 2019 Published online: July 31, 2019 